\title{
ENQUÊTE SUR LES EFFETS VÉCUS AU COURS DE L'ACTIVITÉ BIOGRAPHIQUE: VERS UNE PERSPECTIVE MICRO-PHÉNOMÉNOLOGIQUE POUR PENSER L'HERMÉNEUTIQUE DU SOI
}

\section{- HERVÉ BRETON}

Université de Tours

RÉSUMÉ L'étude des « effet éprouvés » par le sujet proposée dans cet article vise à tracer des perspectives pour avancer vers une compréhension qualitative, expérientielle et phénoménale, de ce qui est vécu et de ce qui se transforme au cours du travail narratif à dimension biographique. L'activité biographique implique le sujet selon deux plans: l'épreuve du travail narratif en première personne, la formation de soi qui résulte de l'expérience du comprendre. Ces deux plans font l'objet d'un examen à partir de l'analyse de quatre procédés: saisie de l'expérience, description de moments, mise en intrigue, relecture et historicisation. Il est conduit à partir d'une démarche dite micro-phénoménologique qui ouvre des perspectives inédites pour l'émergence de nouvelles connaissances dans le domaine de l'herméneutique et de la formation de soi.

Mots-clés : Biographie. Expérience. Formation. Narration. Micro-phenomenologie.

\section{ABSTRACT INQUIRY ON THE EXPERIENTIAL EFFECTS OF THE BIOGRAPHICAL ACTIVITY: TOWARDS A MICRO- PHENOMENOLOGICAL WAY TO UNDERSTAND THE HERMENEUTICS OF THE SELF}

The study of the "experienced effects" for the adult proposed in this article aims to draw out perspectives for progress towards a qualitative, experiential and phenomenal understanding of what is lived and transformed in the course of narrative work with a biographical dimension. The biographical activity involves the person in two ways: the experience of narrative work in the first person, the transformation that results from the experience of understanding. These two plans are examined on the basis of an analysis of four processes: accessing to the experience, describing moments, story composi- 
tion, re-reading and historicization. It is based on a so-called micro-phenomenological approach that opens up new perspectives for the emergence of new knowledge in the field of hermeneutics and andragogy.

Keywords: Biography. Experience. Learning. Narrative. Micro-Phenomenology.

\section{RESUMO ESTUDO SOBRE OS EFEITOS VIVIDOS DURANTE A ATIVIDADE BIOGRÁFICA: POR UMA PERSPECTIVA MICROFENOMENOLÓGICA DA HERMENÊUTICA DE SI}

O estudo dos "efeitos experienciados" pelo sujeito, proposto neste artigo, visa a traçar caminhos que permitam avançar na direção de uma compreensão qualitativa, experiencial e fenomenal do que é vivido e do se transforma, ao longo do trabalho narrativo de dimensão biográfica. A atividade biográfica implica o sujeito sob dois planos: o esforço do trabalho narrativo, na primeira pessoa, e o da formação de si-mesmo, resultante da experiência do compreender. Esses dois planos são objeto de uma análise realizada mediante quatro procedimentos: apreensão da experiência, descrição de momentos vividos, composição do enredo, releitura e historicização. Procedese, com base numa abordagem dita microfenomenológica, que abre perspectivas inéditas para a emergência de novos conhecimentos no âmbito da hermenêutica e da formação de si.

Palavras-chave: Biografia. Experiência. Formação. Narração. Microfenomenologia.

Différents paradigmes sont agissants dans ce qu'il est possible d'appeler les " sciences de la formation "'. L'un d'entre eux pense les démarches biographiques en lien avec une dynamique de formation, via laquelle le sujet accède à des formes d'historicité par la mise en sens temporelle du vécu, sa mise en mots et sa mise en récit. Selon cette perspective, les procédés mobilisés au cours du travail narratif ont des effets métamorphiques qui relèvent

1 La revue Savoirs (2017/1, $\left.n^{\circ} 13\right)$ a consacré un numéro à l'étude des conditions d'émergence des «sciences de la formation». Le lecteur pourra consulter, par exemple, l'article de Jean-Pierre Boutinet intitulé: «Des sciences de la formation peuvent-elles exister et avec quelles spécificités épistémologiques?». d'une dynamique de formation de soi. C'est le cas pour les courants des histoires de vie en formation (PINEAU \& LEGRAND, 2007) et des recherches biographiques en éducation (DELORY-MOMBERGER, 2015) qui s' inscrivent dans un paradigme compréhensif, s'originant dans les traditions de la Bildung, de la Lebensphilosophie, de l'herméneutique de soi.

Une question est alors ouverte: elle concerne la compréhension des processus au cours desquels le récit de vie participe d'une formation de soi. L'enquête porterait ici sur les effets expérientiels du travail narratif et sur les modalités par lesquelles il concourt à la mise en mouvement d'une dynamique formative 
chez le sujet qui s'y exerce, voire, si l'activité est conduite au sein d'un collectif, comme cela est le cas dans les approches via les Histoires de vie en formation, de dynamiques de coformation. Il s'agira dans cet article de tracer des perspectives, pour avancer vers une compréhension qualitative, expérientielle et phénoménale, de ce qui est vécu et de ce qui se transforme au cours de l'activité biographique. Pour ce faire, la méthode initiée analyse les procédés narratifs mobilisés dans les démarches biographiques, puis interroge les effets vécus par le sujet lorsque lesdits procédés sont employés. Ces procédés, au nombre de quatre (l'éveil du souvenir, la description du vécu, la composition du récit, la relecture) sont dans un premier temps décrits à partir des approches provenant de la phénoménologie descriptive - ou micro-phénoménologie. Ils sont ensuite pensés, à la fois dans leur qualité processuelle, expérientielle, et formatrice.

\section{Modes de donation du vécu et dimensions expérientielles de l'activité biographique}

La mise en récit dont il est question au cours de l'activité biographique s'instaure à partir de l'expérience du sujet, est conduite par le sujet lui-même, et produit des effets éprouvés qui transforment ses modes d'existence. La dynamique de formation au cours de ces démarches s'accomplit dans un mouvement par lequel le sujet saisit son expérience pour l'examiner à partir de moments singuliers, puis, dans un temps second, selon une perspective longitudinale. Ce travail de saisie est réalisé « en première personne », ce qui lui confère sa dimension expérientielle. Ce point est central: examiner son expérience fait advenir à la conscience des contenus (les souvenirs) qui, en se présentant à elle, produisent des effets. L'examen de l'activité réflexive mobilisée au cours du tra- vail biographique peut donc s'intéresser, à ce stade, à deux dynamiques: celle des modalités par lesquelles les souvenirs adviennent à la conscience; celle des modalités par lesquelles ces souvenirs produisent un effet expérientiel et transformateur pour le sujet.

\section{Les modalités par lesquelles le souvenir se donne à la conscience}

L'expérience vécue dans le cours de la vie se présente d'abord au sujet sur le mode de l'immédiateté. La rétention des impressions éprouvées dans le présent vivant s'opère à son insu, par synthèse passive (BEGOUT, 2000), sans recours au volontaire ${ }^{2}$. En d'autres termes, les modes de configuration par lesquels les vécus se trouvent retenus et associés restent très largement imperçus et non thématisés. Capté par l'activité quotidienne, accaparé par la conduite de sa vie, le sujet se trouve immergé dans les contenus mondains. C'est pourtant à partir de ces contenus qui sédimentent en mémoire que s'initie l'activité biographique, notamment lors de sa première phase: celle de la "saisie de l'expérience ». Interroger des vécus devenus "souvenirs » (instants, moments, périodes de vie) suppose d'opérer dans l'après-coup, par retour réflexif, des gestes qui consistent à se «tourner vers ", à "s'intéresser (de nouveau) » à des moments passés pour les saisir thétiquement.

\section{Les modalités par lesquelles le souvenir produit un effet pour le sujet}

Avant d'aller plus loin dans l'examen des procédés visant la saisie de contenus expérientiels déposés en mémoire, nous pensons né-

2 Le lecteur pourra consulter les ouvrages de Paul Ricœur, Soi-même comme un autre (1990) et Le volontaire et l'involontaire (1950/2009) dans lesquels le thème de «l'activité sans agent » fait l'objet d'études approfondies. 
cessaire de définir les raisons qui fondent la proposition suivante: saisir et réfléchir son expérience produit un effet pour la personne qui s'y adonne. C'est en effet (entre autres) via la clarification de ces effets que les dimensions formatrices de l'activité biographique peuvent être pensées de manière qualitative. Deux types d'effets peuvent être différenciés: " les effets que cela fait » (NAGEL, 1974) et « les effets que cela génère ». L'étude des « effets que cela fait » porte ici sur la dimension expérientielle (l'éprouvé) du travail narratif. Penser des vécus singuliers, situés dans le passé, fait vivre " au présent » des émotions, sensations, impressions... Le constat est banal. Cependant, nous pensons nécessaire d'étudier ces effets vécus afin de caractériser et de définir qualitativement la dimension expérientielle des démarches biographiques.

Ces effets peuvent être directement associés aux vécus examinés. Ils sont alors considérés comme constitutifs du souvenir. Le fait, par exemple, que je me souvienne que j'ai rencontré ma compagne sur le bord du Gange, en Inde, m'évoque, lorsque le souvenir se présente (et même si cela fait plus de vingt ans maintenant), des impressions et perceptions associées à ce moment particulier d'un matin de mars 1994, sur les ghats de Bénarès. Par cet exemple, il est donc possible de considérer que le fait de saisir un moment passé dans son histoire produit des effets vécus au présent. Une première approche est de considérer que les effets vécus au contact du souvenir se donnent au sujet sur le mode de l'inattendu et de l'inédit. Penser son vécu comporte ainsi une dimension "périlleuse ", du fait que ce qui se donne à la conscience se découvre, voire s' impose au sujet. Il faut faire avec et prendre acte de ce qui s'est constitué en « matière expérientielle » dans le cours de l'existence, et qui se donne comme contenu à la conscience lorsque le souvenir se présente. Ainsi, exami- ner un vécu comportant des formes de rupture de continuité (transition, exclusion, deuil...) fait vivre des impressions qui peuvent ne pas être attendues et qui, advenues dorénavant à la conscience, en occupent le champ.

Ce constat nous conduit à la seconde acception des " effets que cela fait »: ceux que l'activité biographique génère dans le temps. Il s'agit ici d'un second type d'effet, non associé au contenu du vécu sédimenté (les impressions agrégées au souvenir), mais résultant de l'éprouvé survenu lors de l'accès au souvenir tel qu'il s'est présenté à la conscience. Il s'agit là d'un point décisif: l'activité biographique produit des effets vécus, qui forment expérientiellement (PINEAU, 1991). Soit: les compréhensions survenant au cours de l'activité biographique sont des expériences en soi dont la puissance est transformatrice. Il s'agit alors d'examiner ce qu'est " l'expérience du comprendre ». Ce point fait l'objet d'une étude minutieuse par Zahavi (2015), à partir notamment d'un exemple proposé par Strawson (1994): "Strawson nous demande de considérer la situation dans laquelle Jacques (un Français monolingue) et Jack (un Anglais monolingue) sont tous deux en train d'écouter les mêmes actualités en français. Jacques et Jack n'ont certainement par la même expérience, car seul Jacques est capable de comprendre ce qui est dit; seul Jacques est en possession de ce que nous pourrions appeler une expérience de compréhension. Pour le dire autrement, cela produit normalement un certain effet que cela fait, sur le plan expérientiel, de comprendre une phrase. Il y a une différence expérientielle entre le fait d'entendre une phrase que l'on ne comprend pas, et le fait de l'entendre en la comprenant » (ZAHAVI, 2015, p. 87). En prolongeant l'idée contenue dans cet exemple, il est possible de considérer que l'examen des expériences passées, dans leur singularité ou dans les liens qu'elles entretiennent entre 
elles, dans l'ordre et la place qu'elles tiennent dans l'histoire de vie, cela génère des compréhensions qui sont éprouvées « expérientiellement »: cela fait quelque chose de comprendre des événements advenus au cours de son histoire. Les effets ici sont cependant différents de ceux associés au souvenir: se remémorer et comprendre sont deux expériences qualitativement distinctes. Le vécu de compréhension est une expérience durant laquelle le point de vue porté sur un moment et le rapport qu'entretient ce moment avec d'autres dans la trame narrative se transforment graduellement et qualitativement. Cette transformation du rapport à son vécu agit ainsi potentiellement sur trois plans pour le sujet: de manière rétrospective (par transformation du point de vue qui configure l'expérience passée), dans le présent vivant (changement qualitatif des modes d'existence), de manière prospective (métamorphose des perspectives de sens). Il est dons possible d'interroger « l'effet que cela fait » de vivre une expérience métamorphique.

\section{L'herméneutique du soi et les procédés de l'activité narrative}

Dans son article devenu célèbre, "What is the effect to be a bat ? ", Thomas Nagel a mis au jour le « problème difficile » de la conscience, qui peut être résumé de la manière suivante: $\mathrm{s}^{\prime}$ il est relativement possible de percevoir ce que provoque pour moi une expérience, les modalités par lesquelles ces effets se donnent me restent " opaques ». Nagel propose, pour sa démonstration, de considérer que la chauve-souris vit des expériences, et que ces expériences produisent, de son point de vue, un effet qui est simplement vécu. La question qui résulte du problème exposé est alors la suivante: comment procéder pour comprendre la manière par laquelle, selon la perspective de la chauve-souris, se « constitue un point de vue » singulier sur le monde éprouvé et habité. La question est moins complexe du point de vue humain car l'exploration du vécu trouve le soutien d'un langage doté d'une fonction narrative (VICTORRI, 2002). Toutefois, pour certains aspects, le problème reste entier: les humains vivent des effets lorsqu'ils vivent des expériences, sans être en capacité de savoir ni connaître les raisons qui font que les événements vécus provoquent tels types d'effets plutôt que d'autres. Ce point peut sembler simple lorsque l'expérience vécue concerne, par exemple, la dégustation d'une tarte au citron: je peux sans difficulté remarquer le goût sucré-acidulé présent en bouche, la densité et la dimension croquante de la pâte, le velouté suave de la crème... Cependant, je ne saurais dire ce qui me fait privilégier ces aspects de l'expérience aux dépens d'autres, comme par exemple, le goût de la pâte, ou la douceur de la fine gelée qui recouvre la crème... En bref, l'expérience se donne à moi selon des modes qui adviennent avec la force de l'évidence, sans que ces modes de donation puissent faire l'objet d'un examen thématique. Les mêmes processus sont vécus au cours de l'activité biographique: les événements vécus (les souvenirs d'événements, de rencontres, de discussion, d'orientation...) se donnent eux aussi à la conscience selon différents aspects qui s'imposent au sujet sans que leurs modes de donation puissent, dans un premier temps, ouvrir la voie à un travail thématique. Autrement dit, du fait de la transparence des modes de donation, l'attention du sujet est captée par le contenu des vécus qui produisent leurs effets sur le mode de l'évidence et de l'immédiateté au sujet. Cette captation masque et rend opaque le rapport entretenu avec les événements survenus au cours de son existence. Selon cette perspective, le travail biographique se présente comme l'occasion d'une déprise et d'une ouverture des possibles dans 
les manières d'appréhender les événements survenus au cours de l'existence. C'est ce point que nous proposons d'examiner au cours de la prochaine section: étudier les modes de donation des effets vécus au cours de l'activité biographique. Nous le faisons à partir d'un essai d'explicitation de quatre procédés qui nous semblent y être à l'œuvre: la saisie de l'expérience (se tourner vers); la description de moments singuliers; la composition des récits; la relecture des récits.

\section{Modes d'accès au souvenir et "saisie de l'expérience "}

L'expérience peut être pensée différemment selon qu'elle est vécue sur le mode de l'immédiateté ou dans l'après-coup. S' interroger sur l'expérience " déjà-vécue », c'est s'intéresser (de nouveau) à des moments passés pour les saisir comme " thème ", comme « objet d'examen ». Ce travail consistant à se " tourner vers » nécessite pour être compris dans son déroulement, de définir ce qui perdure, sédimente et se constitue (ou se construit) dans le cours de l'expérience. Cette perspective a fait l'objet de publications récentes dans le domaine de la formation ${ }^{3}$. Comment procéder alors, pour se «tourner vers son vécu »? L'expression peut être comprise comme une démarche, initiée par le sujet, dans laquelle une attention est portée à des expériences passées. Ce « devenir attentif » mobilise des gestes qui, pour être réalisés, supposent d'en développer une forme de maîtrise: il s'amorce

3 Nous pouvons citer ici les trois numéros d'Education Permanente, intitulés " Construire l'expérience ", avec pour thèmes: « Réflexivité et pratique professionnelle » ( $\left.n^{\circ} 196\right)$; « Travail et développement professionnel » ( $n^{\circ}$ 197); « Formation expérientielle et intelligence en action » ( $n^{\circ}$ 198). Parmi les publications récentes: Barbier, R et Thievenaz, J. (2013). Le Travail de l'expérience. Paris: L'Harmattan; Albarello, L., Barbier, J-M., Bourgeois, E., et Durand, M. (2013). Expérience, activité, apprentissage. Paris: PUF. Zeitler, A., Guérin, J., Barbier, J-M. (2012). La construction de l'expérience. Recherche \& Formation, 70. en effet via des formes de ralentissement, qui initient des processus de déprise permettant de se défocaliser des contenus qui occupent la conscience afin qu'un espace s'ouvre, afin que les souvenirs adviennent. En d'autres termes, devenir attentif aux vécus passés génère des transformations qualitatives de l'expérience temporelle, dans le présent vivant. Apprendre à ralentir, se proposer à soi de prendre le temps (ERICKSON, 1998), accepter de vivre des formes de lenteur, peut être considéré comme le premiers pas d'une pratique réflexive à visée narrative. Vient ensuite une série de gestes qui relèvent d'une micro-dynamique de l'éveil (DEPRAZ, 2014a) du souvenir. Ils jalonnent en effet des passages allant du vécu sédimenté en mémoire à l'évocation de cette expérience passée, puis de sa saisie réflexive en vue d'une première mise en mots. Associés aux gestes visant à initier des formes de ralentissement dans le cours du présent vivant, sont donc conduits des gestes assimilés au maintien d'un espace d'ouverture, propice à ce que le regard puisse se porter sur les vécus et que s'amorce l'exploration de l'expérience.

\section{L'exploration des moments et des strates du vécu}

Nous avons, à ce stade, cherché à différencier les effets éprouvés au contact de vécus sédimentés (les souvenirs) des modes de donation par lesquels ces vécus se donnent à la conscience, et donc, se donnent à vivre au sujet. Nous avons ensuite émis l'idée que les modes de donation pouvaient être analysés à partir de l'étude des « effets que cela fait » de revivre un moment passé, ceci indépendamment du contenu expérientiel du moment lui-même. Cette distinction entre contenu du vécu, et modes de donation des effets vécus du fait de la reprise de contact avec ce contenu situé, est de premier ordre pour penser le 
travail d'exploration et de description de moments singuliers.

Le procédé d'exploration peut être envisagé à partir d'une série de gestes venant interroger l'expérience en vue de sa mise en mots. Ces gestes varient cependant en fonction de la durée de référence du vécu saisi. Appréhender une expérience passée pour la réfléchir, c'est la définir et la contenir dans un périmètre pour l'examiner. Ce point est déterminant pour l'analyse de l'exploration: la saisie de l'expérience et l'exploration de son contenu sont deux procédés co-dépendants. Selon l'empan temporel du vécu de référence, les gestes et niveaux d'exploration varient. Nous rencontrons ici la thèse de Ricœur, qui met au jour la réciprocité des processus de temporalisation de l'expérience avec ceux de la mise en récit au cours de l'activité narrative: «Mon hypothèse de base est à cet égard la suivante: le caractère commun de l'expérience, qui est marqué, articulé, clarifié par l'acte de raconter sous toutes ses formes, c'est son caractère temporel. Tout ce qu'on raconte arrive dans le temps, prend du temps, se déroule temporellement; et ce qui se déroule dans le temps peut être raconté. Peut-être même tout processus temporel n'est-il reconnu comme tel que dans la mesure où il est racontable d'une manière ou d'une autre. Cette réciprocité supposée entre narrativité et temporalité est le thème de « Temps et récit » (RICEEUR, 1986, p. 14). S' interroger sur les processus réciproques entre « temps et récit » fait ainsi émerger un champ d'étude: celui des liens unissant, dans les textes ou discours, la durée des vécus évoqués, exprimés ou narrés, et les niveaux de profondeur atteints dans l'exploration et la description. Nous suggérons ici un rapport, une dépendance, entre la vitesse des récits et les matériaux expérientiels mis au jour dans les expressions orales ou écrites. Nous approchons alors deux notions: le niveau de détail de la description du vécu; la pluralité des aspects pris en compte pour son expression. L'idée est ici la suivante: la description de moments courts permet de décrire en détail différents aspects du vécu, tandis que la narration de longues périodes s'intéressera principalement aux enchaînements des faits. Cela conduit à interroger le troisième procédé: celui des modes de composition, qui intègrent dans une histoire, des vécus de plus ou moins longues durées, pour les associer ensemble à mesure que s'édifie la trame narrative du récit.

\section{La composition des récits: mise \\ en mots, mise en sens, mise en intrigue}

L'activité narrative suppose de saisir son expérience, de prêter attention (procédé 1), et de l'explorer (procédé 2) par temporalisation et mise en mots. Ces deux premiers procédés peuvent être considérés comme préparatoires au travail de composition des récits, qu'ils soient oraux ou écrits. Composer consiste, dans une démarche biographique, à configurer dans un récit différents moments et événements en les associant ensemble pour faire advenir l'histoire d'une vie, ou d'une période de vie. Soit, en d'autres termes, de tenir ensemble des moments singuliers ce qui, du fait de la composition réalisée, fait émerger une unité de sens qui les relient logiquement et temporellement. Il y a donc, dans le mouvement qui s'amorce par la mise en mots et qui perdure dans la mise en sens, une dynamique de transformation qualitative du rapport entretenu entre les événements du point de vue du sujet. Cette transformation résulte de compréhensions qui permettent de renouveler les possibilités d'interprétation des moments, transitions, rencontres... survenues dans le cours de la vie.

C'est par cette transformation qualitative du rapport à son histoire que le sujet peut 
vivre les expériences de compréhension précédemment évoquées. Ricœur, dans son ouvrage "Temps et récit, Tome 1 », propose une analyse détaillée des procédés de composition à partir du chapitre VI de la Poétique d'Aristote. Il fait de la « mise en intrigue » le pivot d'une dynamique qui consiste à associer et tenir ensemble des moments vécus dans une histoire. Cette dynamique peut être comprise à partir de deux processus: le rapprochement et l'association. La composition dans le récit récit procède d'un travail qui consiste à relier des événements dans une histoire, ces liens s'établissant, du point de vue du sujet, selon un principe de concordance: les associations qui sont produites le sont du point de vue du sujet, selon des rapprochements qui lui apparaissent vraisemblables. La composition suppose donc de structurer des critères - ou de les interroger si ceux-ci sont déjà à l'œuvre -, afin que, du point de vue su narrateur, advienne un sentiment, un ressenti, un effet, qui permettent de tenir pour vraies les associations produites. Autrement dit, du point de vue du sujet, l'histoire, pour apparaître cohérente, doit prendre la forme du véritable. Ricœur (1983, p. 80), à partir de la Poétique d'Aristote, identifie trois traits en lien avec le principe de concordance: complétude, totalité, étendue appropriée. Pour que l'intrigue apparaisse vraisemblable, le récit doit intégrer une durée de vécu suffisante. Ce périmètre d'exploration identifié (l'étendue), le narrateur doit alors faire émerger une logique de déroulement, qui procède, par identification, d'une succession d'événements qui construisent la trame du récit. Le troisième trait - celui de la complétude - porte alors sur la cohésion d'ensemble. Il y a donc ici conjuguées pour la composition, une dimension chronologique - celle du déroulement temporel propre à la succession - et une dimension logique caractérisée par le processus d'association
L'identification des procédés de composition du récit initie donc, par le travail qu'elle suppose, une transformation qualitative du regard porté par le sujet sur son expérience. Composer, c'est en effet tenir pour vrai des associations et des liens advenus dans le cours de l'existence. Ou, nous l'avons dit, c'est initier une « logique » entre des moments du vécu et ainsi faire advenir " une intrigue » au sein du chronologique. La configuration agit ici comme une intensification, une modification de la dimension étale du temps. Et, résultant du travail configurant, la composition du récit fait événement par les compréhensions qui résultent des associations générées: " Le comprendre ne s'adresse donc pas à la saisie d'un fait mais à l'appréhension d'une possibilité d'être » (RICEEUR, 1986, p. 101). Le travail de composition, bien que portant sur des événements passés, produit des effets vécus au présent. Différentes questions s'ouvrent: quels sont les effets vécus résultant de l'expérience du « comprendre »? Comment penser la temporalité du comprendre ? Comment appréhender la réciprocité entre la gradualité de la composition et les processus de compréhension? L'expérience du comprendre peut-elle être décrite de manière micro-phénoménologique? Les recherches ici envisagées portent sur l'analyse des effets vécus, en première personne, au cours de l'activité narrative, au gré des étapes qui s'initient à partir de la mise en mots pour faire advenir à l'horizon de la conscience des points de vue inédits, des manières transformées de penser sa vie et ses modes d'existence.

\section{La relecture: historicisation et}

\section{mise en perspective}

Le quatrième procédé proposé à l'étude est celui de la relecture. Il consiste à porter un regard sur l'expérience configurée dans le récit, que celui-ci prenne la forme d'un discours ou 
d'un texte. Surgit alors la question de la référence: tout discours ou récit s'édifie sur un ou des vécus en première personne qui constituent le socle de la mise en mots et de la mise en intrigue. Ce travail d'édification procède cependant, nous l'avons noté précédemment, d'une transformation qualitative à l'issue de laquelle l'expérience de référence - ou le vécu de référence, selon les termes de Pierre Vermersch - fait advenir un monde: « Référence et horizon sont corrélatifs comme le sont la forme et le fond. Toute expérience à la fois possède un contour qui la cerne et la discerne, et s'enlève sur un horizon de potentialités qui en constituent l'horizon interne et externe: interne en ce sens qu'il est toujours possible de détailler et de préciser la chose considérée à l'intérieur d'un contour stable; externe en ce sens que la chose visée entretient des rapports potentiels avec tout autre chose sur l'horizon d'un monde total, lequel ne figure jamais comme objet de discours » (RICEUR, 1983, p. 147). La relecture procède ainsi, par la mise en question de la dimension configurée du récit, d'une double interrogation: celle portant sur la pertinence de l'étendue du récit, celle portant sur le niveau de fermeté de l'intrigue. Ce travail de relecture fait vivre lui-même des effets: celui d'une potentielle contestation de l'histoire et du monde advenus par le discours: " le discours est toujours au sujet de quelque chose: il se réfère à un monde qu'il prétend décrire, exprimer ou représenter; l'événement, [...)] c'est la venue au langage d'un monde par le moyen du discours » (RICEUR, 1986, p. 116).

Comment, dès lors, envisager ici les procédés de relecture? Ils interrogent les chaînes d'association produites durant la phase de composition, ceux du périmètre d'exploration (pertinence des moments retenus pour la mise en intrigue), ceux des modalités de saisie des vécus (qualité de l'évocation et niveau de détail de la description). L'examen propre à la relecture croise ainsi les dimensions chronologique et logique. Les travaux de Baudouin (2010) portant sur les régimes cinétiques ${ }^{4}$ de textes autobiographiques donnent à penser, par une étude en troisième personne, ces éléments. L'identification des facteurs de ralentissement ou d'accélération du « temps narré dans le texte » le mène à proposer en mobilisant les travaux de Genette (1972), quatre modalités de composition du récit:

Tableau 1: Procédés narratifs et variations cinétiques du récit (BAUDOUIN, 2010, p. 419)

\begin{tabular}{|l|l|l|}
\hline Pause & Action suspendue & $\begin{array}{l}\text { Important facteur } \\
\text { de ralentissement }\end{array}$ \\
\hline Scène & Action narrée & $\begin{array}{l}\text { Facteur de } \\
\text { ralentissement }\end{array}$ \\
\hline Ellipse & Action omise & $\begin{array}{l}\text { Facteur } \\
\text { d'accélération } \\
\text { d'accélération }\end{array}$ \\
\hline
\end{tabular}

Ce tableau différencie quatre modes de composition du récit en fonction de leur effet cinétique: la " pause » dont la caractéristique est de suspendre le déroulement et d'accueillir une séquence de description plus ou moins détaillée; la " scène » qui tient à égalité de temps le récit descriptif et le déroulement de l'histoire; le " sommaire » dont la fonction est d'opérer la conjonction entre les différentes séquences du texte. Ce dernier tient ensemble la scène et "l'ellipse », qui constitue un temps occulté de l'histoire. Ces quatre « figures de composition " constituent des critères pour entrer dans l'analyse de la trame temporelle des récits. Cette analyse peut, de manière complémentaire, intégrer les dimensions qualitatives des vécus considérés (en prenant en

4 Baudouin (2010, p. 413) définit « les régimes d'économie cinétique d'un texte » comme "Le "rapport" entre une quantité chronologique et un nombre de caractères ». 
compte, par exemple, les niveaux d'exploration), puis interroger le niveau de fermeté des associations produites qui viennent fonder la logique du texte. À partir de ces différents points, il est possible de penser les procédés de relecture comme un travail d'interrogation des dimensions configurées du récit, par exploration et creusement de périodes et de phases de l'histoire. Cela conduit le narrateur à interroger les associations tenant les moments et événements ensemble, à rouvrir le périmètre de l'exploration des moments (GALVANI, 2011), à détailler de manière plus fine des successions advenues dans le cours de la vie.

\section{Les effets de l'activité narrative et leur pouvoir transformateur}

La formalisation de quatre procédés a été effectuée afin de caractériser, par des gestes et des actes, l'activité biographique réalisée au cours de sessions de formation intégrant le travail aux histoires de vie, aux ateliers d'écritures à visée autobiographique. Ce travail de description et de définition étant réalisé, une analyse/synthèse des effets vécus du point de vue du sujet impliqué dans le travail narratif est maintenant possible. Chacun des procédés peut faire l'objet d'un examen particulier; l'étude peut également porter sur les effets résultant de l'accomplissement des quatre procédés, pour en penser les effets. Rappelons-le, cet examen vise à mieux comprendre les dimensions expérientielles et formatrices de la narration en contexte biographique. L'hypothèse émise ici est que l'analyse de ces effets, dans le temps, est de nature à définir et préciser les formes de l'expérience du comprendre et les métamorphoses qu'elles génèrent dans les modes d'existence du sujet. L'analyse des effets éprouvés suppose de les faire passer au langage, soit de disposer d'une méthode permettant, non pas de décrire des contenus expérientiels (les moments d'une histoire), mais les modes de donation de cette histoire au sujet. Cela suppose de porter attention aux impressions, perceptions et inférences (les effets) advenant au cours de la conduite de l'activité biographique: perceptions de tâtonnement lors de la recherche des moments, dynamiques d'attente au cours de l'activité de composition, prises de sens lors de la relecture du récit, transformation des manières de concevoir les enchaînements logiques entre différentes périodes de vécus... Ces différents processus peuvent être pensés comme des conséquences et/ou des résultats de l'activité biographique. Nous supposons qu'ils sont vécus par le sujet sans pour autant qu'ils soient remarqués, ni qu'ils fassent l'objet d'un travail de thématisation.

\section{Analyse des effets expérientiels éprouvés lors de la "saisie » de l'expérience}

Les effets vécus associés à cette phase relèvent de formes de ralentissement éprouvés qui sont potentiellement transformatrices de la manière de vivre le temps. S'interroger sur son vécu, tourner son regard vers l'expérience passée, cela suppose de vivre des formes de suspens qui sont expérientiellement vécues et ressenties sous la forme d'un relâchement, d'une déprise. Le registre de description des effets éprouvés durant cette phase de l'activité narrative relève donc du domaine de l'attention, décrite par Depraz comme une « capacité à ne pas se fixer, à tenir l'ouverture d'une indécision » (DEPRAZ, 2014a, p. 98). Une compréhension plus fine de ce qui est « expériencé » concernera ici les dynamiques d'éveil, d'attention, d'ouverture, en visant leur expression à partir de perceptions immédiates advenant lorsqu'un contenu expérientiel est saisi sur le mode noématique. Adviennent également des 
effets associés au passage de l'expérience au langage. L'exploration micro-phénoménologique, en modifiant les horizons de perception, découvre des dimensions de l'expérience restées imperçues du fait de la centration du sujet sur des aspects saillants du vécu qui captent son attention. Ce découvrement résulte d'un éveil attentionnel aux différentes strates de vécus précédemment évoquées. Il s'opère potentiellement en avance par rapport au langage. La découverte de dimensions imperçues de l'expérience révèle, de manière associée, qu'il faudra alors combler pour que des aspects inédits du vécu éprouvé trouvent à se dire dans le récit.

\section{Analyse des effets résultant des procédés de l'exploration du vécu}

L'expression de ces dimensions du vécu apparaît alors, dans un temps premier, comme floue et vague. Ainsi, le travail micro-phénoménologique sollicite par inférence un travail d'invention langagière favorisant le passage de l'expérience au langage. L'analyse de l'effet produit pour le narrateur peut donc s'intéresser aux ressentis liés à la transformation de la manière d'appréhender l'expérience passée, du fait de sa mise en mots. Différents éléments peuvent alors être notés: constitution d'un sol pour la mise en récit, appropriation d'un vocabulaire permettant l'expression du vécu selon différents aspects et différentes temporalités, élargissement des horizons de perception... Ces éléments participent d'une transformation de la manière de voir et de penser les moments appréhendés au cours de l'activité biographique, par temporalisation, aspectualisation, élucidation.

L'expérience du comprendre résulte ainsi de la circulation du regard, lors de l'activité d'exploration, entre trois modes d'appréhension de l'expérience:
- Le contenu du vécu, qui réfère à la situation concrètement éprouvée, dans son ambiance, son déroulement, ses faits marquants, ses actions concrètes, sa matérialité.

- Ce qui est perçu de la situation, et notamment de ce qui se détache sur fond d'objets et d'impressions restant en arrière-plan. S'initie alors un premier examen des modes de donation de l'expérience, par conscientisation du « relief expérientiel » associé au souvenir: objets saillants qui captent l'attention, éléments expérientiels faisant l'objet d'une cécité, dimensions du vécu restant imperçues.

- Ce qui est ressenti lors de l'accès au souvenir: impressions, sensations, inférences. Ces éléments révèlent et témoignent du rapport entretenu par le sujet avec les moments décrits, qu'ils se constituent en périodes de vie, en phases d'une transition advenue au cours de l'existence, ou en moments singuliers.

Cette théorie des niveaux différencie ainsi ce qui relève du contenu de l'expérience perçu par les sens (le noématique), ce qui relève de la perception et du relief expérientiel dans les modes de donation (contenus se présentant sur le mode de l'évidence, venant capter l'attention et qui, pour être examiner, suppose de s'en déprendre). Selon ce modèle, l'activité biographique accompagne et génère un détachement graduel de l'emprise du contenu de l'expérience et favorise, du point de vue su sujet qui pense son histoire, la circulation des modes d'appréhension du vécu, entre examen du contenu, réflexivité sur les effets éprouvés lors de l'exploration, analyse des formes de configuration générées pour le récit de soi. En d'autres termes, le fait de circuler entre différents niveaux d'exploration du vécu au cours 
de l'activité biographique, entre contenu de l'expérience et élucidation des modes de donation, génère des expériences de compréhension qui font socle pour les processus de formation de soi.

\section{Analyse des effets résultant des procédés de composition du récit de vie}

Les dimensions expérientielles de l'activité de composition du récit de vie éprouvée en première personne peut être examinée selon deux axes: la mise en ordre chronologique des événements advenus au cours de l'existence; la mise en sens logique de ces événements selon les principes de l'association et du vraisemblable. L'analyse des effets vécus doit porter ici sur le contenu phénoménal, subjectif et expérientiel, se donnant au sujet lorsqu'il met en lien des événements lui apparaissant préalablement sur le mode du disparate et de l'hétérogène. L'étude est ici complexe, car le travail de composition prend du temps, sa durée s'étendant de quelques heures à quelques mois. Par où commencer alors? Les effets vécus alternent entre les phénomènes d'attente, d'anticipation, d'éveil, de prise de sens, et mobilisent des formes d'inférences à la fois transductives et abductives ${ }^{5}$ (DENOYEL, 1999). Les impressions associées vont de plus varier en fonction du contenu noématique de l'expérience mise au jour durant la phase d'exploration et de description. Deux grands types de

5 Les inférences transductive et abductive fonctionnent sans référence au connu et à l'habituel. L'inférence transductive procède par tâtonnements et rapprochements entre des éléments expérientiels qui apparaissent sur le mode de l'hétérogène et de l'éparse. La transduction opère dans le vague et l'incertain (CHAUVIRE, 1995). L'abduction relève d'une prise de sens aboutissant à une compréhension devenant significative (2010) et rendant intelligibles des phénomènes auparavant ressentis comme étranges, problématiques ou énigmatiques. Cette compréhension une fois réalisée, devient une ressource possible de l'action, un savoir pouvant être remobilisé au gré des situations de manière plus ou moins régulière. vécus peuvent être, de manière un peu grossière, différenciés: ceux relevant d'une perception de désorientation, du fait de la complexité même de la composition qui réinterroge, chemin-faisant, le sens déjà-là par lequel se pense l'existence; ces perceptions se donnent alors au sujet sur le mode de la perte, de la fragilité, voire du péril: la mise en question de la cohérence de la trame narrative, qui fait vivre des sensations et perceptions de trouble, de doute, parfois de deuil. Le second type d'effet, par contraste, résulte non de la dissociation mais de l'association. Il est perçu sur le mode du « conjoindre » et du " comprendre ». Cette expérience se donne du fait des processus d'historicité initiés: ils constituent une dynamique de transformation par laquelle le point de vue porté par le sujet sur les événements de son histoire change, se transforme et s'émancipe du tenu pour vrai, et par là même, s'aventure dans des « voies de signification inédites $»$.

\section{Analyse des effets résultant des procédés de relecture du récit de vie}

La relecture du récit peut adopter plusieurs formes: relecture concrète du texte autobiographique, voire des versions successives produites lors de la composition du récit; expression orale qui suppose de sélectionner des passages du récit; expérience de la réception lors des phases de socialisation au sein d'un collectif (lors des sessions d'histoires de vie, par exemple), ce qui a pour effet d'interroger, en première et seconde personne (DEPRAZ, 2014b), les modes de composition du récit de soi. Les procédés de relecture peuvent donc prendre des tournures pluridimensionnelles et s'initier selon des temporalités qui s'échelonnent du proche immédiat à l'après-coup (de l'ordre de quelques semaines, voire plusieurs 
mois). Les effets peuvent dont être considérés comme diffus, en advenant selon un processus de maturation pouvant succéder au travail de composition: ils peuvent prendre la forme d'une mise en question des associations logiques tenant ensemble les événements et aspects du récit, d'une transformation du regard porté sur les événements narrés, d'un changement de régime quant aux énonciations possibles de l'histoire vécue.

\section{Analyse des effets résultant de l'ensemble de la démarche}

Il est également possible d'interroger les effets vécus à l'échelle des quatre procédés. La démarche consiste ici à penser l'activité biographique dans sa dynamique interne, par conjugaison de l'ensemble des procédés mis au jour. Il s'agit moins de procéder selon une arithmétique que de penser la résonance des effets entre eux: ralentissement, ouverture, éveil, ralentissement émotionnel, ouverture des horizons de perception, transformation qualitative des manières de voir... L'appréhension de ces processus dans la durée d'un parcours suppose de constituer une épistémologie, croisant l'étude micro-phénoménologique des effets vécus avec les démarches d'enquête portant sur les modes d'existence (SOURIAU, 2009; LATOUR, 2012), en vue d'une formalisation des effets expérientiels et transformateurs de l'activité biographique en formation d'adultes.

\section{La phénoménologie}

\section{expérientielle comme science du vécu en première personne}

À ce stade, nous nous heurtons - il faut en convenir - à une difficulté d'ordre méthodologique. Les effets que nous avons cherché à analyser et thématiser sont en effet assez peu décrits qualitativement. Le format de cet écrit ne le permet pas tout à fait. Cependant, la principale difficulté ne réside pas là. C'est ici que les travaux de la phénoménologie husserlienne, et plus précisément une des branches actuelles de la phénoménologie dite « pratique » ou expérientielle (DEPRAZ, 2009) - ou micro-phénoménologie - ouvrent des perspectives d'exploration qui intéressent notre étude. Quels sont ses enjeux, sinon de structurer des approches permettant l'examen des contenus expérientiels, des effets associés à cette forme d'enquête, la mise au jour des modes de donation du vécu ? Nous voyons ici se dessiner un champ de recherche singulier pour les travaux consacrés à l'activité biographique: celui d'un analyse qualitative et expérientiel des activités d'appréhension du vécu et des formes de compréhension que cela génère. Cet examen pour porter par exemple sur différentes strates de cette activité: à quoi « je » suis sensible (processus affectif), à quoi « je » suis attentif (dynamique attentionnelle); ce que «je » me dis lorsque « je » perçois, délibère, décide (processus cognitifs et langagiers); ce que «j' » attends et anticipe (dynamique temporelle) ... Et via la phénoménologie descriptive (DEPRAZ, 2013), s'ouvrent des perspectives de recherche en vue d'une " science de l'expérience vécue » (Petitmengin, Bitbol, Ollagnier-Beldame, 2015) à partir d'une perspective épistémologique « en première personne » (DEPRAZ, 2013; ZAHAVI, 2005). En d'autres termes, la micro-phénoménologie ouvre des voies de recherche, dans la continuité des travaux de Francisco Varela $(1989,1993)$ sur l'énaction, factrices de nouvelles connaissances sur les dimensions expérientielles et formatrices du travail biographique, lors des sessions d'histoires de vie en formation, par exemple (PINEAU \& LEGRAND, 1993; LAINE, 2004). L'étude des modes de manifestation de l'expérience pour le sujet qui les éprouve constitue pour ces recherches un point nodal. Ils résultent en effet de l'histoire 
vécue et/ou héritées, tout en restant agissants dans le présent vivant.

Pour examiner le vécu sur un plan expérientiel, la fondation de méthodes ouvrant droit à sa description, en première ou seconde personne constitue un enjeu de premier plan. Le travail de description peut porter sur les « effets vécus » (PETITMENGIN, 2010), au cours de l'activité biographique, au cours de ses phases et procédés. Il ne va pas de soi. Sans méthode stabilisée, l'étude du vécu emprunte les voies de l'introspection versant dans l'internalisme ou le mentalisme. Ou à l'inverse, nous l'avons dit, elle s'en tient à une science en troisième personne, expérimentale, réduisant l'activité du sujet à ce qui peut en être observé. L'avancée vers une troisième voie, créant les conditions possibles d'une étude rigoureuse de l'expérience vécue au cours des démarches procédant du "récit de soi », nécessite donc de structurer des approches en première personne déployées à partir de quatre critères essentiels: viser des vécus singuliers, guider l'exploration vers les strates pré-réfléchies de l'expérience ${ }^{6}$, régler le niveau de détail de la description selon différentes échelles temporelles ${ }^{7}$, orienter la description vers des aspects particuliers du vécu (sens, inférence, langage, impressions...).

\section{Comprendre les dimensions} expérientielles de l'activité

\section{biographique via l'enquête micro- phénoménologique}

Nous avons cherché, dans cet article, à structurer des repères pour la structuration d'un champ de recherche visant, à partir des

6 Le lecteur pourra sur ce point consulter l'article de C. Petitmengin (2010); signalons l'article « Description et vécu » de Pierre Vermersch publié dans la revue en ligne Expliciter ( $n^{\circ} 89$, mars 2011).

7 Nous signalons sur ce point l'article de F. Lesourd paru dans la revue Expliciter ( $n^{\circ} 46$, octobre 2002), intitulé « Des fenêtres attentionnelles temporelles. » approches micro-phénoménologiques, la connaissance des dimensions expérientielles et transformatrices de l'activité biographique en formation d'adultes. Nous avons, pour ce faire, détaillé les procédés mobilisés au cours de cette activité, puis cherché à en comprendre les effets successivement, puis de manière dynamique, selon deux plans: expérientiels et transformateurs. Les deux régimes narratifs - la phénoménologie descriptive et l'herméneutique de soi - sont dans notre enquête conjugués et pensés comme complémentaires: le travail herméneutique est envisagé comme une technique de soi (FOUCAULT, 2002) faisant advenir des formes d'historicité pour le sujet. Il se trouve ainsi au fondement d'approches qui pensent le récit selon une perspective anthropoformatrice. Différents courants, dans les domaines de la recherche en sciences humaines et sociales, ainsi que dans les secteurs de la formation professionnelle, de la formation continue et de la formation pour adultes, mobilisent les pratiques narratives en vue d'apprentissages qui procèdent de l'émancipation de croyances ou de discours hérités, d'une mise en sens de soi, d'une redéfinition des perspectives de sens, d'une reconnaissance des acquis expérientiels. L'activité narrative devient ici une pratique de formation contemporaine, consubstantielle au fait même d'exister, les mondes humains se caractérisant notamment par leur condition biographique (DELORY, 2010).

L'apport de la phénonoménologie descriptive, ou micro-phénoménologie, réside dans le fait que les effets vécus et transformateurs des démarches biographiques peuvent être concrètement décrits, de manière expérientielle, à partir d'une perspective en première personne. Vivre les effets du travail narratif ne garantit pas que ces effets pourtant éprouvés puissent être décrits par la personne qui les a vécus. Le travail micro-phénoménologique ouvre des 
perspectives inédites, permettant de mettre au jour ces effets perçus, ressentis, de les décrire et de les mettre en mots, d'en analyser la force et le potentiel transformateur. C'est donc vers la mise en mots de ce qui se donne à vivre au cours de l'activité biographique que nous proposons de mobiliser les approches micro-phénoménologiques: impressions associées au souvenir, qualité expérientielle des processus de compréhension, transformation qualitative des manières de voir et de se percevoir, changements d'ambiance dans les modes d'existence, transformation des manières d'habiter le monde.

\section{Bibliographie}

Baudouin, J-M. De l'épreuve autobiographique. Berne: Peter Lang, 2010.

Bégout, B. La généalogie de la logique. Paris: Vrin, 2010.

Breton, H. Interroger les savoirs expérientiels via la recherche biographique. Revue Le sujet dans la Cité, n. 8, p. 25-41, 2017.

Chauviré, C. Peirce et la signification. Introduction à la logique du vague. Paris: PUF, 1995.

Chauviré, C. Ogien, A. La régularité. Habitude, disposition et savoir-faire dans l'explication de l'action. Paris: Editions EHESS, 2002.

Delory-Momberger, C. La condition biographique. Paris: Téraèdre, 2010.

Delory-Momberger, C. De la recherche biographique en éducation. Fondements, méthodes, pratiques. Paris: Anthropos, 2015.

Denoyel, N. Alternance tripolaire et raison expérientielle à la lumière de la sémiotique de Peirce. Revue française de pédagogie, n. 128, p. 35-42, 1999.

Depraz, N. Plus sur Husserl. Une phénoménologie expérientielle. Paris: Atlande, 2009.

Depraz, N. D'une science descriptive de l'expérience en première personne: pour une phénoménologie expérientielle. Studia Phaenomenologica, 13 (1), p. 387-402, 2013.

Depraz, N. Attention et vigilance. Paris: PUF, 2014a.

Depraz, N. (Dir.). Première, deuxième, troisième personne. Bucarest: Zeta Books, 2014b.

Erickson, M. Ma voix t'accompagnera. Paris: Hommes et groupes, 1998.

Foucault, M. L'herméneutique du sujet. Cours au Collège de France, 1981-1982. Paris: Seuil; Gallimard, 2001.

Galvani, P. Moments de formation et mise en sens de soi. Paris: L'Harmattan, 2011.

Genette, G. Figures III. Paris: Seuil, 1972.

Lainé, A. Faire de sa vie une histoire. Paris: Desclée de Brouwer, 2004.

Latour, B. Enquête sur les modes d'existence. Une anthropologie des modernes. Paris: La Découverte, 2012.

Nagel, T. Mortal questions. London: Cambridge University Press, 2012 [1979].

Petitmengin, C. La dynamique pré-réfléchie de l'expérience vécue. Alter, n. 18, p. 165-182, 2010.

Petitmengin, C., Bitbol, M., Ollagnier-Beldame, M. Vers une science de l'expérience vécue. Intellectica, v. 2, n. 64, p. 53-76, 2015.

Pineau, G. Formation expérientielle et théorie tripolaire de la formation. In : Courtois, B.; Pineau, G. (Eds.). La formation expérientielle des adultes $\mathrm{Pa}$ ris: La Documentation Française, 1991. p. 29-40.

Pineau, G. Legrand, J-L. Les histoires de vie. Paris: PUF, 1993.

Ricœur, P. Temps et récit. 1. L'intrigue et le récit historique. Paris: Seuil, 1983.

Ricœur, P. Du texte à l'action. Paris: Seuil, 1986.

Souriau, E. Les différents modes d'existence. Paris: Presses Universitaires de France, 2009.

Strawson, G. Mental reality. Cambridge: MIT 
Press, 1994.

Varela, F. Autonomie et connaissance. Paris: Seuil, 1989.

Varela, F., THOMPSON, E., ROSCH, E. L'inscription corporelle de l'esprit. Paris: Seuil, 2003.

Victorri, B. Homo narrans: le rôle de la narration dans l'émergence du langage. Langages, 146 (L'origine du langage), p. 112-125, 2002.
Zahavi, D. Subjectivity and selfhood. Investigating the first-personn perspective. London: MIT Press, 2005.

Zahavi, D. Intentionnalité et phénoménalité. Un regard phénoménologique sur le problème difficile. Philosophie, n. 124, p. 80-104, 2015.

Recebido em: 28.01.2018

Aprovado em: 10.03.2018

Hervé Breton est Maître de conférences, EE1 EES, Université de Tours. Président de l'Association Internationale des Histoires de Vie en Formation et de Recherche Biographique en Education. Responsable du Diplôme universitaire Histoires de vie en formation (DU HIVIF). e-mail : herve.breton@univ-tours.fr

Université de Tours, 3 rue des Tanneurs, 37000 Tours. Tel : $0033 .(0) 671.740 .006$ 